\title{
The Metabolic Changes in Fresh Versus Old Stored Blood Used in Priming of Extracorporeal Circuit in Cardiopulmonary Bypass for Pediatric Patients - First Results
}

\author{
Normunds Sikora, Valts Ozolins, Lauris Smits, Elina Teivane, Inta Bergmane, Aris Lacis \\ Children's University Hospital, Pediatric Cardiology and Cardiosurgery Clinic
}

\begin{abstract}
Summary
Introduction. One of the main goal in modern pediatric bypass equipment is to reduce the size of the extracorporeal circuit in order to minimize the prime volume. High priming volume can produce a low hematocrit on CPB in small infants, which results in electrolyte imbalance and decreased tissue oxygenation, therefore the donor blood should be used in the prime.

The use of donor blood itself has several disadvantages, including complement activation, induction of a transfusion reaction, infusion of lactate, glucose and potassium, therefore this should be avoided as much as possible. When blood is stored, many alterations occur in its constinuents, in particular an increase in potassium and lactate levels, and decrease in $\mathrm{pH}$, which have been associated with severe complications.
\end{abstract}

Aim of the study. To evaluate the effect of length of storage of packed red blood cells (PRBC) on the concentration of potassium, sodium, lactate, glucose and pH in PRBC used in pediatric cardiac surgery.

Materials and methods. From 0ctober,2006 until June,2009 blood samples were drawn from 78 PRBC used in cardiopulmonary bypass before they were added to the priming solution. All PRBC were splited in two groups depending on the age of blood: 5 days $\geq$ (Group 1), $\mathrm{n}=44$ and 5 days< (Group 2), $\mathrm{n}=34$. Blood samples were analyzed by GEM PREMIER 3000.

Results. Seventy eight PRBC used in pediatric cardiac surgery were included in the study. In 44 blood samples the age of PRBC was under 5 days (Group 1) and in 34 blood samples the age of PRBC was over 5 days (Group 2). The mean storage time of PRBC in Group 1 was $4+/-1$ days and in Group $2-8+/-3$. The pH value in Group 1 was higher than in Group $2(6.7+/-0.1$ vs $6.5+/-$ $0.2, \mathrm{P}=0.000859)$. There was a significant difference between both groups in terms of potassium level $(6.1+/-1.8 \mathrm{vs} 9.4+/$ 2.6, $P<0.0001)$ and lactate level $(7.2+/-1.4$ vs $10.1+/-1.7, P<0.0001)$, but no significant difference in terms of sodium level $(138.3+/-3.9$ vs $135.6+/-4.7, P=0.056688)$ and glucose level $(346.5+/-36.1$ vs $336.3+/-50.7, P=0.509321)$. There was an intermediate correlation in both groups in terms of storage time and level of potassium, storage time and level of lactate, $\mathrm{pH}$ and level of potassium, pH and level of lactate, and the levels of potassium and lactate. There was a linear increase in the levels of potassium and lactate depending on the storage age of PRBC. Conclusions.It is absolutely crucial to use as fresh packed red blood cells as possible in pediatric cardiac surgery to avoid such unexpected complications as transfusion-induced cardiac arrest due to hyperkalaemia. There are also other metabolic changes such as hyperlactemia and low pH, should be avoided, when performing pediatric cardiopulmonary bypass. The research is going to be continued, possibly starting to evaluate the levels of potassium and acid-base balance, when circulating the priming solution for some time before the initiation of cardiopulmonary bypass.

Key words: packed red blood cells; cardiopulmonary bypass.

\section{INTRODUCTION}

One of the main goal in modern pediatric bypass equipment is to reduce the size of the extracorporeal circuit in order to minimize the prime volume, which can actually exceed the blood volume of a neonate by as much as $200 \%$ to $300 \%$, whereas in an adult patient, the priming volume accounts for only $25 \%$ to $30 \%$ of the blood volume. Even though nowadays there has been developed smaller tubing and oxygenators, which reduces priming volume significantly, it is still an issue in pediatric cardiopulmonary bypass (CPB). High priming volume can produce a low hematocrit on CPB in small infants, which results in reduction of plasma proteins and clotting factors, decrease of the colloid osmotic pressure (interstitial edema), electrolyte imbalance, exaggerated release of stress hormones with activation of complement, white blood cells and platelets, therefore it is compulsory to use the donor blood in the prime. The use of donor blood itself has several disadvantages, including transmission of viral particles, complement activation, induction of a transfusion reaction, infusion of lactate, glucose and potassium, and citrate-phosphate-dextrose infusion, therefore this should be avoided as much as possible. However, for the reason of hemodilution most institutions use packed red blood cells in their priming solutions (4).

Although packed red blood cells (PRBC) are essential to maintain appropriate hematocrit level and adequate oxygen delivery in children, they are associated with significant metabolic imbalances (acid-base, glucose, 
electrolyte), leading to a number of severe complications. When blood is stored, many alterations occur in its constinuents, in particular an increase in potassium and lactate levels, and decrease in $\mathrm{pH}$. The most important changes from the physiological range pointed out by the Ratcliffe JM et al in their research are in sodium, potassium, glucose and lactate concentration. They experienced increase in the level of potassium and lactate, and decrease in the level of sodium and glucose. Besides they pointed out a strong correlation between the duration of storage and the level of sodium, potassium and lactate (7). The biggest problem appears to be hyperkalaemia. Because red blood cell membranes are only slightly permeable to potassium, their movement is largely dependent on energy-dependent transport mechanisms (glycolytically derived adenosine triphosphate). During storage, red blood cell membranes age, adenosine triphosphate synthesis and potassium pumping decrease, and intracellular potassium leaks into the supernatant, which leads to hyperkalaemia (9). There has been reported cases of mortality in neonates during transfusion of stored PRBC, mainly because of hyperkalaemia-induced cardiac arrest, even of a relatively fresh 6 -day old blood unit $(1,3)$. Hyperkalaemia related to transfusion depends also on volume and rate of PRBC administration. It was shown that transfusion-associated cardiac arrest may develop with rapid administration of PRBC even with modest transfusion volume (9). Besides, as there is no oxygen in PRBC, the energy is mainly produced in the way of anaerobic glycolysis, therefore there is an increase in the level of lactate and decrease in the level of glucose in the supernatant leading to acidosis $(4,5,8)$.

Hyperkalaemia and hyperlactatemia related to transfusion itself is not a big problem. What makes it an important issue in pediatric cardiac surgery are many other mechanisms contributing to the risks of PRBC transfusion-induced hyperkalaemia or increased potassium cardiotoxicity include hyperglycemia, hypocalcemia, hypothermia and acidosis. First, surgical stress and shock are associated with hyperglycemia, which induces an increase in serum osmolality causing potassium to exit cells. Second, massive transfusion of citrated blood is associated with hypocalcemia, which predisposes to cardiac membrane instability at lower potassium levels. Hypothermia also slows the metabolism of citrate, which exacerbates hypocalcemic states. Third, in hypothermia heart becomes more sensitive to the toxic effects of potassium (9). Therefore many institutional protocols advocate limiting the use of $\mathrm{PRBC}$ in priming solution for the pediatric CPB to relatively fresh stored PRBC to avoid such complications.

\section{AIM OF THE STUDY}

To evaluate the effect of length of storage of PRBC on the concentration of potassium, sodium, lactate, glucose and $\mathrm{pH}$ in PRBC used in pediatric cardiac surgery needed for cardiopulmonary bypass in Children's University Hospital, Clinic for Pediatric Cardiology and Cardiac Surgery.

\section{MATERIALS AND METHODS}

From October, 2006 until June,2009 blood samples were drawn from 78 PRBC used in cardiopulmonary bypass before they were added to the priming solution. Fresh PRBCs were preferred and patients would receive old ones only if no fresh PRBC was available. All PRBC were splited in two groups depending on the age of blood: 5 days $\geq$ (Group 1), $\mathrm{n}=44$ and 5 days $<$ (Group 2), $\mathrm{n}=34$. The groups were made according to the mean age of PRBCs available in the blood bank of our hospital for pediatric cardiac surgery. Blood samples were drawn from the $\mathrm{PRBC}$, stored at $4^{\circ} \mathrm{C}$ in preservative solution, consisting of citrate, dextrose, phosphate and adenine (CP2 and SAGM ), and were analyzed by GEM PREMIER 3000. Comparisons between corresponding variables at different times in each group were carried out by using the Student $\mathrm{t}$ test. There were calculated correlation index between different levels of electrolytes and the age of PRBC.

\section{RESULTS}

Seventy eight PRBC used in pediatric cardiac surgery were included in the study. In 44 blood samples the age of PRBC was under 5 days (Group 1) and in 34 blood samples the age of PRBC was over 5 days (Group 2). The mean storage time of PRBC in Group 1 was $4+$ /- 1 days and was significantly shorter compared with the storage time in Group 2 ( $8+/-3$ days, $\mathrm{P}=0.000264)$. The $\mathrm{pH}$ value in Group 1 was higher than in Group 2 (6.7 +/0.1 vs $6.5+/-0.2, \mathrm{P}=0.000859)$. There was a significant difference between both groups in terms of potassium level $(6.1+/-1.8$ vs $9.4+/-2.6, \mathrm{P}<0.0001)$ and lactate level $(7.2+/-1.4$ vs $10.1+/-1.7, \mathrm{P}<0.0001)$, but no significant difference in terms of sodium level (138.3 $+/-3.9$ vs $135.6+/-4.7, \mathrm{P}=0.056688)$ and glucose level $(346.5+/-36.1$ vs $336.3+/-50.7, \mathrm{P}=0.509321)(T A B L E$ 1).

Table 1. Mean values +/- SD of different parameters in both groups

\begin{tabular}{|c|c|c|c|}
\hline & $\begin{array}{c}\text { Goup l } \\
\mathrm{n}=44)\end{array}$ & $\begin{array}{c}\text { Group 2 } \\
(\mathrm{n}=34)\end{array}$ & P value \\
\hline $\begin{array}{c}\text { Storage } \\
\text { time }\end{array}$ & $\begin{array}{c}4+/- \\
1\end{array}$ & $\begin{array}{c}8+/- \\
3\end{array}$ & 0.000264 \\
\hline $\mathrm{pH}$ & $\begin{array}{c}6.7+/- \\
0.1\end{array}$ & $\begin{array}{c}6.5+/- \\
0.2\end{array}$ & 0.000859 \\
\hline Potassium & $\begin{array}{c}6.1+/- \\
1.8\end{array}$ & $\begin{array}{c}9.4+/- \\
2.6\end{array}$ & $<0.0001$ \\
\hline Sodium & $\begin{array}{c}138.3+/- \\
3.9\end{array}$ & $\begin{array}{c}135.6+/- \\
4.7\end{array}$ & 0.056688 \\
\hline Glucose & $\begin{array}{c}346.5+/- \\
36.1\end{array}$ & $\begin{array}{c}336.3+/- \\
50.7\end{array}$ & 0.509321 \\
\hline Lactate & $\begin{array}{c}7.2+/- \\
1.4\end{array}$ & $\begin{array}{c}10.1+/- \\
1.7\end{array}$ & $<0.0001$ \\
\hline
\end{tabular}

There was an intermediate correlation in both groups in terms of storage time and level of potassium (correlation coefficient 0.42 vs 0.24 ) (Figure 1 and Figure 2), storage time and level of lactate (correlation coefficient 0.45 vs 
0.6) (Figure 3 and Figure 4), $\mathrm{pH}$ and level of potassium (correlation coefficient 0.49 vs 0.77 ), $\mathrm{pH}$ and level of lactate (correlation coefficient 0.61 vs 0.55 ), and the levels of potassium and lactate (correlation coefficient 0.61 vs 0.6$)$. There was a linear increase in the levels of potassium and lactate depending on the storage age of PRBC (Figure 1, Figure 2, Figure 3, Figure 4).
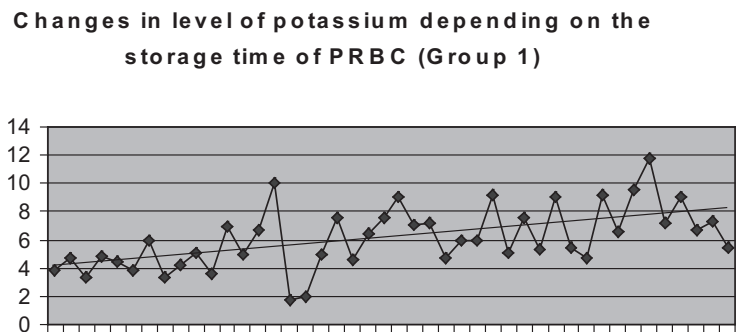

Storage time of PRBC (days)

Changes in level of lactate depending on the storage time of PRBC (Group 1)

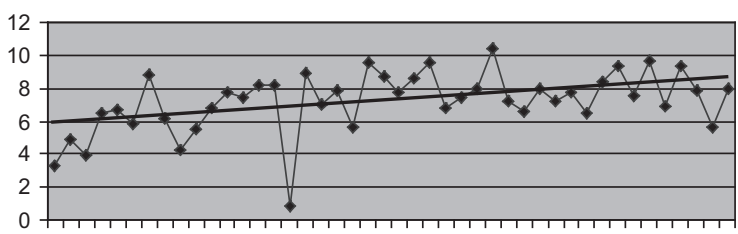

Storage time of PRBC (days)

Changes in level of potassium depending on the storage time of PRBC (Group 2)

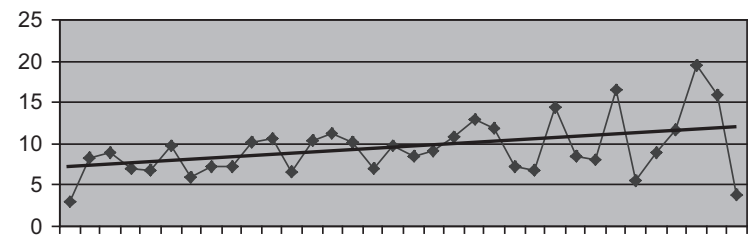

Storage time of PRBC (days)

Changes in level of lactate depending on the storage time of PRBC (Group 2)

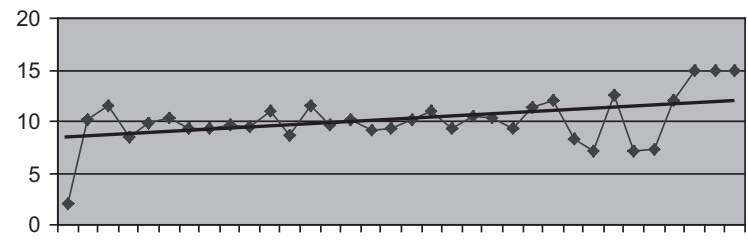

\section{DISCUSSION}

As this is compulsory to use RPBC for priming the extracorporeal circuit in pediatric cardiac surgery to maintain the adequate hematocrit and oxygenation, it is very important to have as fresh PRBC as possible, because fresh ones are presumably more balanced metabolically than stored PRBCs. They contain less potassium and lactate, more glucose, and higher $\mathrm{pH}$. It is an important issue in pediatric cardiac surgery due to many other mechanisms contributing to the risks of PRBC, such as surgical stress, shock, hypothermia.

One of our major concerns with prolonged storage was excess potassium in the red cell supernatant, which could cause cardiac problems. There has been reported cases of mortality in neonates during transfusion of stored PRBC, mainly because of hyperkalaemia-induced cardiac arrest, even of a relatively fresh 6-day old blood unit $(1,3)$. What is more, in neonatal surgery, the stored blood may be irradiated, if there is a possibility of impaired imunity, which may cause a further increase in potassium levels $(5,10,11)$. There have been a number of researches showing a significant increase in potassium in old PRBCs $(5,7,9,10,11)$. Our results clearly shows that potassium level was significantly higher in old stored PRBCs, and there was an intermediate correlation in both groups in terms of storage time and level of potassium. The other papers shows that there is an increase in the level of lactate and decrease in the level of glucose in the supernatant leading to acidosis due to the energy mainly being produced in the way of anaerobic glycolysis $(4,5,8)$. We experienced the same increase in concentrations of lactate and decrease of $\mathrm{pH}$, and an intermediate correlation in both groups in terms of storage time and level of lactate. Besides there was also an intermediate correlation between the levels of potassium and lactate, which made us think that all metabolical processes taking place in PRBCs during the storage could be conjoint. What is the most important thing what we can see in our results is a linear increase in the levels of potassium and lactate depending on the storage age of PRBC, therefore it is compulsory to use as fresh PRBCs as possible in pediatric cardiac surgery. Our other results shows only a slight decrease in the level of sodium and glucosis during storage, similarly stated by Ratcliffe JM et al in their research (7).

Storage time of PRBC (days) 


\section{CONCLUSIONS}

In conclusion, our findings shows that it is absolutely crucial to use as fresh packed red blood cells as possible in pediatric cardiac surgery to avoid such unexpected complications as transfusion-induced cardiac arrest due to hyperkalaemia. Besides there are other metabolic changes such as hyperlactemia and low $\mathrm{pH}$, should be avoided, when performing pediatric cardiopulmonary bypass. The research is going to be continued, possibly starting to evaluate the levels of potassium and acid-base balance, when circulating the priming solution for some time before the initiation of cardiopulmonary bypass, or the change of quality of PRBCs according to the age.

\section{Conflict of interest: None}

\section{REFERENCES}

1. Bazz EM, Kanazi GE, Mahfouz RA,Obeid MY. An unusual case of hyperkalaemia-induced cardiac arrest in a pediatric patient during transfusion of a "fresh" 6-day-old blood unit // Transfus Med, 2002; $12(6): 383-6$

2. Fleming JM, Remenapp RT, Bartlett RH, Annich GM. Hyperkalemia of the blood-primed ECLS circuit does not result in post-initiation hyperkalemia in infants // Perfusion, 2006; 21 (3):173 - 177

3. Hall TL, Barnes A, Miller JR, et al. Neonatal mortality following transfusion of red cells with high plasma potassium levels // Transfusion 1993; 33(7):606 - 9

4. James Jaggers, Ian R. Shearer, Ross M. Ungerleider Cardiopulmonary bypass in infants and children // In: Gravlee P.Glenn, Richard F.Davis, Mark Kurusz, Joe R.Utley. Cardiopulmonary bypass. 2nd ed. Philadelphia: Lippincot Williams\&Wilkins; 2000; $214-264$
5. Keidan I, Amir G, Mandel M, Mishali D. The metabolic effects of fresh versus old stored blood in the priming of cardiopulmonary bypass solution for pediatric patients // J Thorac Cardiovasc Surg 2004; $127(4): 949-52$

6. Liu J, Ji B, Feng Z, et al. The effect of preprocessing stored red blood cells on neonates undergoing corrective cardiac surgery // ASAIO J 2007; $53(6): 680-3$

7. Ratcliffe JM, Elliot MJ, Wyse RK, et al. The metabolic load of stored blood. Implications for major transfusions in infants // Arch Dis Child 1986; 61:1208-1214

8. Schroeder TH, Hansen M. Effects of fresh versus old stored blood in the priming solution on whole blood lactate levels during pediatric cardiac surgery // Perfusion 2005; 20(1):17 - 9

9. Smith HM, Farrow SJ, Ackerman JD, et al. Cardiac arrests asociated with hyperkalemia during red blood cell transfusion: a case series // Anesth Analg 2008; 106:1062 - 9

10. Swindell CG, Barker TA, McGuirk SP, et al. Washing of irradiated red blood cells prevents hyperkalaemia during cardiopulmonary bypass in neonates and infants undergoing surgery for complex congenital heart disease // Eur J Cardiothorac Surg 2007; 31(4):659- 64

11. Vohra HA, Adluri K, Willets R, et al. Changes in potassium concentration and haematocrit associated with cardiopulmonary bypass in paediatric cardiac surgery // Perfusion 2007; 22(2): 87-92

\section{Address:}

Normunds Sikora

Pediatric Cardiology and Cardiosurgery Clinic, Children's Clinical University Hospital Vienibas gatve 45, Riga, LV-1004, Latvia e-mail: normundssikora@yahoo.com 